\section{Optimierung von Abwasseranlagen}

Die Abwasserentsorgung steht vor einer Vielzahl von Herausforderungen, die sich gleichermaßen auf die Abwasserableitung und die Abwasserbehandlung beziehen. Dabei geht es in dieser Ausgabe der Österreichischen Wasser- und Abfallwirtschaft um die Optimierung von Abwasseranlagen im engeren Sinne, nämlich um die energetische Optimierung von Abwasserreinigungsanlagen, im weiteren Sinne um die Optimierung des Betriebs der Entwässerungssysteme und um Optimierungsansätze für Gewässerschutzanlagen zur Behandlung der Abwässer von Verkehrsflächen.

Die Verbesserung der Energieeffizienz von Kläranlagen ist mittlerweile ein traditionelles Thema, wenn es um die Optimierung von Abwasseranlagen geht. Das Ziel, eine „energieautarke“ Kläranlage zu schaffen, taucht in immer mehr Arbeitspapieren und Konsulentenaufträgen auf. Die Kläranlage als Energiezentrale scheint zum „Kampfruf“ gegen den Klimawandel und als wirtschaftlicher Hoffnungsschimmer für die budgetgepeinigten Kommunen geworden zu sein. Die Autoren des ersten Beitrages zeigen in einem Detailreichtum und in aller Vielfalt die Analyse, Identifikation und die Umsetzungsmöglichkeiten der Energieeinsparung auf Abwasserreinigungsanlagen in sehr praktischer ingenieurtechnischer Weise.

Neben planerischen Aufgaben der Siedlungsentwässerung gewinnen betriebliche Aufgaben wie z. B. die Kanalinspektion immer größere Bedeutung. Schon seit geraumer Zeit ist unbestritten, dass der Kanalinspektion in Hinblick auf den Betrieb (insbesondere für die Reinigung) eine vorrangige Rolle zukommt. Dies wurde auch im ÖWAV-Regelblatt 43 „Optische Kanalinspektion“ betont, in dem klar zwischen der optischen Inspektion für den betrieblichen Überblick und jener für eine detaillierte bauliche Aufnahme unterschieden wird. Die erstgenannte Inspektion wird laut den Untersuchungsergebnissen der Autoren des zweiten Beitrags infolge der technologischen Entwicklung der letzten Jahre bald ein unverzichtbarer Bestandteil der Optimierung des Kanalbetriebs sein.

Der dritte Beitrag behandelt ein immer wichtiger werdendes Thema im Zuge der Umsetzung der Wasserrahmenrichtline in Bezug auf die erforderliche Behandlung von Oberflächenabflüssen, insbesondere von Verkehrsflächen. Ausgehend von der Annahme, dass viele Mikroverunreinigungen partikulär gebunden sind und daher durch Elimination der absetzbaren Stoffe eine große Wirkung erzielt werden kann, fallen die Erfahrungen bei der Überprüfung dieser Wirkung bei Gewässerschutzanlagen leider negativ aus.

Ein Optimierungspotenzial ist bei diesen Abwasseranlagen, von denen in nicht allzu ferner Zukunft in Österreich wahrscheinlich noch sehr viele gebaut werden müssen, von der Planung über die Umsetzung bis zum Betrieb der Anlagen gegeben.

Der Herausgeber ist überzeugt, dass die hier präsentierten Ergebnisse und Erfahrungen auf breites Interesse innerhalb der Fachwelt stoßen und für die zukünftigen abwassertechnischen Entwicklungen relevant sind.

\section{Univ.-Prof. DI Dr. T. Ertl}

Institut für Siedlungswasserbau, Industriewasserwirtschaft und Gewässerschutz, Department Wasser-Atmosphäre-Umwelt, Universität für Bodenkultur Wien,

Muthgasse 18, 1190 Wien, Österreich

E-Mail: thomas.ertl@boku.ac.at

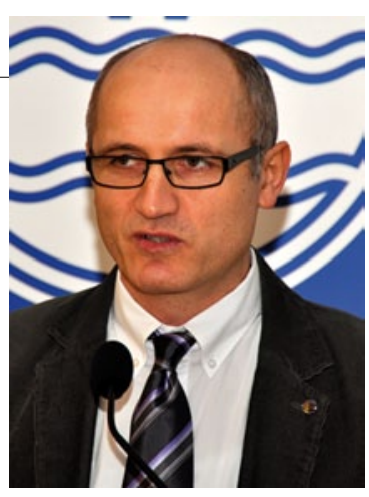

Univ.-Prof. DI Dr. T. Ertl 